\title{
Effects of different polishing methods on color stability of resin composites after accelerated aging
}

\author{
Emine SIRIN KARAARSLAN ${ }^{1}$, Mehmet BULBUL² ${ }^{2}$ Esma YILDIZ ${ }^{3}$, Asli SECILMIS ${ }^{2}$, Fatih SARI ${ }^{2}$ and Aslihan USUMEZ ${ }^{4}$ \\ ${ }^{1}$ Department of Restorative Dentistry, Faculty of Dentistry, Gaziantep University, Gaziantep 27310, Turkey \\ ${ }^{2}$ Department of Prosthodontics, Faculty of Dentistry, Gaziantep University, Gaziantep 27310, Turkey \\ ${ }^{3}$ Department of Pediatric Dentistry, Faculty of Dentistry, Gaziantep University, Gaziantep 27310, Turkey \\ ${ }^{4}$ Department of Prosthodontics, Faculty of Dentistry, Bezmialem Vakif University, Istanbul 34093, Turkey \\ Corresponding author, Emine SIRIN KARAARSLAN; E-mail: karaarslan.emine@gmail.com
}

\begin{abstract}
The purpose of this study was to evaluate the effect of polishing procedures on the color stability of different types of composites after aging. Forty disk-shaped specimens $(\varnothing 10 \times 2 \mathrm{~mm})$ were prepared for each composite resin type (an ormocer, a packable, a nanohybrid, and a microhybrid) for a total of 160 specimens. Each composite group was divided into four subgroups according to polishing method $(n=10)$ : control (no finishing and polishing), polishing disk, polishing wheel, and glaze material. Color parameters $\left(L^{*}, a^{*}\right.$, and $\left.b^{*}\right)$ and surface roughness were measured before and after accelerated aging. Of the polishing methods, glazed specimens showed the lowest color change $\left(\Delta E^{*}\right), \Delta L^{*}$, and $\Delta b^{*}$ values $(p<0.05)$. Of the composite resins, the microhybrid composite showed the lowest $\Delta E^{*}$ value, whereas the ormocer showed the highest $(p<0.05)$. For all composite types, the surface roughness of their control groups decreased after aging $(p<0.05)$. In conclusion, all composite resins showed color changes after accelerated aging, with the use of glaze material resulting in the lowest color change.
\end{abstract}

Keywords: Color stability, Accelerated aging, Composite resins, Surface roughness

\section{INTRODUCTION}

Dental appearance and function of teeth are important to both dentists and patients alike ${ }^{1)}$. While dental function is held in greater regard, aesthetic dental restorative treatments have been shown to wield a positive psychological effect on patients' self-esteem ${ }^{2}$. With increased patient demands for aesthetic restorations, composite resins have widely dominated the field of aesthetic dentistry because of improvements in both physico-mechanical and aesthetic properties. Manufacturers have introduced different shades of restorative materials to fulfill all the requirements for environmental light sensitivity, depth of cure, and color match $^{3)}$.

Matching the initial shade of an uncured composite resin material to that of adjacent natural teeth is a fundamental, and yet extremely challenging, task in aesthetic practices. Once an acceptable match is obtained, the color match should ideally be maintained after polymerization. However, the optical properties of dental composite resins change because of polymerization, and the extent of change is influenced by the brand and shade of resin composites and the wavelength of curing lights ${ }^{4,5)}$. Uchida et $a l .{ }^{6}$ ) also reported that ultraviolet light exposure induced physico-chemical reactions in composite resins, such that lighter shades of composites were likely to be subject to higher color degradation through environmental effects of sunlight exposure.

In the oral environment, many factors can cause the extrinsic discoloration of resin composites: exposure to mouthrinses, consumption of acidic or staining foods and drinks ${ }^{7}$. Intrinsically within the composite resins, structure of the resin matrix and characteristics of the filler particles have a direct impact on surface smoothness and susceptibility to extrinsic staining8). Resin matrix and filler particles of composite resins do not abrade to the same degree due to different degrees of hardness. Surface craters or irregularities then form around hard quartz particles of conventional composite resins after poor finishing and polishing, be it due to techniques and/or instruments. Consequently, these surface irregularities can lead to staining, high wear rates, plaque retention, gingival irritation, and recurrent caries $^{9,10)}$. Conversely, a high degree of smoothness and low surface porosity decrease the adherence of agents responsible for changing the color of composite resins, such as dental biofilms, tobacco, and food colorants ${ }^{11}$. Therefore, it is very important that dental restorations are polished to delay the discoloration and aging processes of composite resins.

Apart from playing a role in the final smoothness of dental composite restorations ${ }^{12}$, the resin matrix also influences color stability. If the matrix is hydrophilic, increased water sorption occurs, resulting in a whiter and more opaque shade ${ }^{13)}$. If the matrix is hydrophobic, it absorbs less water, resulting in greater color stability $^{13,14)}$.

In routine aesthetic procedures, color matching is performed visually. However, environmental lighting conditions influence shade matching and selection. Instrumental color measurements prevail over visual color assessment in terms of overall accuracy and efficiency ${ }^{15)}$ because they provide objective, quantifiable, reproducible, and more rapidly obtainable shade selections ${ }^{16}$. However, in the case of composite resins, 
their color is also influenced by differences in the various shades of resin, curing conditions, resin thickness, and background colors for color measurement ${ }^{17)}$.

Threshold color difference levels based on instrumental color measurements that can be visually perceivable or clinically acceptable have been discussed. The clinically acceptable value for color difference in dental restorative materials is assumed to be $\Delta E^{*} \leq 3.3^{18)}$. In the case of composite resins, accelerated aging reportedly produced significant and perceptible color change $\left(\Delta E^{*}\right)$ of $6.7-27.9^{19)}$, and water aging led to unacceptable color instability and opacity variation ${ }^{11)}$.

The purpose of this in vitro study was to evaluate the effects of different polishing methods on the color stability and surface roughness of four different types of composite resins after accelerated aging. The hypothesis of this study was that the color stability of composite resins would not be affected by different composite types and polishing methods.

\section{MATERIALS AND METHODS}

\section{Preparation of composite resin disk specimens}

Four different types of composites resins (an ormocer, a packable, a nanohybrid, and a microhybrid) of shade A3 and three polishing methods were used (Table 1). For each type of composite resin, 40 disk-shaped specimens were prepared, amounting to a total of 160 disks.

A Teflon plate with a hole $(10 \mathrm{~mm}$ in diameter and $2 \mathrm{~mm}$ in height) was prepared. After a composite resin material was packed into the hole in Teflon plate, a polyester strip was placed on the composite surface to smooth the surface. A glass slide was placed over the polyester strip to ensure excess composite resin material was extruded after pressure was applied and to minimize polymerization reaction inhibition by oxygen $^{20)}$. All composite disk specimens were cured with an LED (light-emitting diode; Demi, Kerr, Danbury, CT, USA) curing light at $1,100 \mathrm{~mW} / \mathrm{cm}^{2}$ for $20 \mathrm{~s}$. Before each curing, light intensity of LED unit was checked using a radiometer (L.E.D. Radiometer, Demetron, SDS/Kerr).

\section{Composite specimen groups according to polishing method}

Specimens of each composite resin were divided into four groups, each containing 10 specimens. In all groups, specimen surfaces were ground flat with 1000-grit silicon carbide paper (Buehler, UK Ltd., Lake Bluff, IL, USA) under running water. For each composite resin, a control group of 10 specimens received no finishing and polishing. The remaining 30 specimens were randomly divided into three groups ( $n=10 /$ group) according to polishing method.

Specimens were polished using one of the following methods:

a) By polishing disk. Using Sof-Lex (3M ESPE, St. Paul, MN, USA), specimens were sequentially polished with medium, fine, and superfine aluminum oxide abrasive disks under running water. b) By polishing wheel. Using Enhance (Dentsply DeTrey, Konstanz, Germany), specimens were polished with wheels and polishing pastes.

c) By glaze material. Specimens were applied with BisCover LV (Bisco, Schaumburg, IL, USA) and cured using an LED (Demi, Kerr, Danbury, CT, USA) curing light at $1,100 \mathrm{~mW} / \mathrm{cm}^{2}$ for $30 \mathrm{~s}$, according to manufacturer's instructions.

Polishing with Sof-Lex and Enhance were performed using intermittent light pressure for $20 \mathrm{~s}$, rinsed, and dried with air/water syringe for $10 \mathrm{~s}$. Abrasive disks and polishing wheels were used with a slow-speed handpiece (Bien-Air Dental SA, Bienne, Switzerland), rotating at approximately 20,000 rpm. After polishing, all specimens were stored in lightproof, dark, plastic boxes in a wet condition.

\section{Instrumental color measurement}

The $L^{*}, a^{*}$, and $b^{*}$ values of composite resin specimens after polishing were measured using a spectrophotometer (Vita Easyshade, Ivoclar Vivadent AG, Schaan, Liechtenstein). All specimens were chromatically measured three times, and then the average values were calculated.

The spectrophotometer consisted of a base unit and a handpiece with a 5-mm-diameter probe tip. Specimen was illuminated with light, and reflected light was analyzed by the spectrophotometer. Color measurement was carried out by placing the probe tip on the central part of specimen, which was placed against a white plate background ${ }^{21-23)}$. To ensure consistency of consecutively repeated color measurements, specimens were positioned at the same place for different color measurements. After color data were collected for each group $(n=10)$, the spectrophotometer was recalibrated. Tip of the handpiece was firmly placed in the calibration port and steadily held in place until the instrument sounded a beep to indicate that calibration was completed.

After baseline color measurement, specimens were subjected to accelerated aging. They were placed on the equipment's fixing plates (Basacryl, Birleşik Akrilik San. Tic. Ltd. Şti., İstanbul, Turkey) and taken to a condensation chamber facing the light source. Aging was carried out using a light and heat aging device (Physical Engineering Department, Gaziantep University, Turkey), which was run for a total exposure time of 58 $\mathrm{h}$ ( $29 \mathrm{~h}$ light on) at a total radiant energy level of 150 $\mathrm{kJ} / \mathrm{m}^{2}$. Aging conditions were as follows - test cycle: 12 min light only, 12 min dark plus back water spray; chamber temperature (panel temperature): $70^{\circ} \mathrm{C}$ light and $30^{\circ} \mathrm{C}$ dark; humidity: 50\% (light) and 90\% (dark); and water temperature: room temperature $\left(22^{\circ} \mathrm{C}\right)$.

After accelerated aging, the $L^{*}, a^{*}$, and $b^{*}$ values of specimens were measured again using the spectrophotometer. The $L^{*}$ coordinate is a measure of lightness-darkness of the specimen: the greater the $L^{*}$, the lighter the specimen. The $a^{*}$ coordinate is a measure of chroma along the red-green axis: a positive $a^{*}$ indicates redness while a negative $a^{*}$ indicates greenness. The $b^{*}$ coordinate is a measure of chroma along the yellow-blue 
Table 1 Composite resins and polishing materials used in this study

\begin{tabular}{|c|c|c|c|}
\hline Composite type/Chemical composition* & Product & Batch No. & Manufacturer \\
\hline $\begin{array}{l}\text { Ormocer } \\
\text { Bis-GMA }^{\text {a }} \text {, di-UDMA } \text {, TEGDMA } \\
\text { three-dimensionally curing } \\
\text { inorganic-organic copolymers, } \\
\text { additive aliphatic and aromatic dimethacrylates, } \\
\text { fillers: glass-ceramic, } \\
\mathrm{SiO}_{2} \text { microparticle } 0.7 \mu \mathrm{m}, 78 \mathrm{wt} \%\end{array}$ & Admira & 937353 & $\begin{array}{l}\text { VOCO Cuxhaven, } \\
\text { Germany }\end{array}$ \\
\hline $\begin{array}{l}\text { Microhybrid } \\
\text { TEGDMA }^{c}, \text { Bis-GMA }{ }^{\mathrm{a}}, \text { BisEMA }^{\mathrm{d}} \text {, UDMA } \\
\text { Zirconia/Silica, particle size } 0.01-3.5 \mu \mathrm{m} \text {, } \\
82 \mathrm{wt} \%, 60 \text { vol } \%\end{array}$ & Filtek Z250 & $8 \mathrm{PR}$ & $\begin{array}{l}\text { 3M ESPE, St. Paul, } \\
\text { MN, USA }\end{array}$ \\
\hline $\begin{array}{l}\text { Nanohybrid } \\
\text { Bis-GMA }^{\mathrm{a}} \text {, TEGDMA }{ }^{\mathrm{c}} \text {, UDMA } \\
\text { glass filler } 1 \mu \mathrm{m} \text {, nanofiller } \mathrm{SiO}_{2} 20-60 \mathrm{~nm} \text {, } \\
87 \mathrm{wt} \%, 71.4 \mathrm{vol} \%\end{array}$ & Grandio & 949138 & $\begin{array}{l}\text { VOCO, Cuxhaven, } \\
\text { Germany }\end{array}$ \\
\hline $\begin{array}{l}\text { Packable } \\
\text { Bis-GMA }^{\text {a }}, \mathrm{UDMA}^{\mathrm{b}} \text {, TEGDMA }{ }^{\mathrm{c}}, \mathrm{BisEMA}^{\mathrm{d}} \\
\text { Zirconia/silica, particle size } 0.01-3.5 \mu \mathrm{m} \text {, } \\
\text { average } 0.6 \mu \mathrm{m}, 61 \text { vol\% }\end{array}$ & Filtek P60 & $9 \mathrm{PE}$ & $\begin{array}{l}\text { 3M ESPE, St. Paul, } \\
\text { MN, USA }\end{array}$ \\
\hline $\begin{array}{l}\text { Polishing disk } \\
\text { Aluminum oxide abrasive disks } \\
\text { (medium, fine, super fine; } \\
20 \text { s for each disk) }\end{array}$ & Sof-Lex & P090508 & $\begin{array}{l}\text { 3M ESPE, St. Paul, } \\
\text { MN, USA }\end{array}$ \\
\hline $\begin{array}{l}\text { Polishing wheels and pastes } \\
\text { Aluminum oxide, } \\
\text { Polymerized urethane dimethacrylate resin, } \\
\text { Aluminium oxide-impregnated wheels and } \\
\text { pastes for } 20 \mathrm{~s} / 10 \mathrm{~s}\end{array}$ & Enhance & 100113 & $\begin{array}{l}\text { Dentsply DeTrey, } \\
\text { Konstanz, Germany }\end{array}$ \\
\hline $\begin{array}{l}\text { Glaze material } \\
\text { Dipentaerythritol diacrylate esters, ethanol }\end{array}$ & BisCover LV & 1000010770 & $\begin{array}{l}\text { Bisco, Schaumburg, } \\
\text { IL, USA }\end{array}$ \\
\hline
\end{tabular}

a: Bisphenol-A-glycidyl methacrylate

b: Diurethane dimethacrylate

c: Triethyleneglycol dimethacrylate

d: Bisphenol A ethoxylated dimethacrylate

*: Information as obtained from manufacturer

axis: a positive $b^{*}$ indicates yellowness while a negative $b^{*}$ indicates blueness ${ }^{17)}$.

The Commission Internationale de l'Eclairage (CIE $L^{*} a^{*} b^{*}$ ) color space was used to determine color differences ${ }^{24)} . \Delta L^{*}, \Delta a^{*}$, and $\Delta b^{*}$ are differences between two colors in the CIE-based color space ${ }^{25)}$. In this study, the colorimetric values of $\Delta L^{*}, \Delta a^{*}$, and $\Delta b^{*}$ were calculated from differences in the respective $L^{*}, a^{*}$, and $b^{*}$ values. The total color difference $\Delta E^{*}{ }_{a b}$ between two colors, each given in terms of $L^{*}, a^{*}$, and $b^{*}$, was calculated from the following formula ${ }^{21)}$ :

$$
\Delta E^{*}{ }_{a b}=\left[\left(\Delta L^{*}\right)^{2}+\left(\Delta a^{*}\right)^{2}+\left(\Delta b^{*}\right)^{2}\right]^{1 / 2}
$$

Surface roughness measurement

Surface roughness was determined using a profilometer
(Surftest Analyzer, Mitutoyo, Tokyo, Japan). Surface roughness was measured in average arithmetic roughness $(R a)$ values. Three measurements were performed for each specimen. The mean value of three measurements for each specimen was used as the $R a$ value of that specimen.

\section{Statistical analysis}

Color stability data were analyzed using two-way ANOVA and Tukey's HSD test $(\alpha=0.05)$. For $R a$ values, paired-samples $t$-tests were used to analyze the data and compare groups. Data were analyzed using a statistical program software, SPSS 13 for Windows (SPSS Inc., Chicago, IL, USA). 


\section{Scanning electron microscope evaluation}

After polishing was completed for each method, the surfaces of specimens were gold sputter-coated to a layer thickness of approximately $60 \AA$ in a vacuum evaporator coater (Quorum SC7620, Quorum Technologies Ltd., West Sussex, UK). Surface topography of specimens was examined under a scanning electron microscope (SEM; JSM-6390LV, JEOL Ltd., Tokyo, Japan) at 5,000× magnification and an accelerating voltage of $20 \mathrm{kV}$.

\section{RESULTS}

Effects of polishing method and composite resin type on color stability

Table 2 shows the two-way ANOVA results for differences in $\Delta E^{*}$ values among the composite resin groups. Polishing method and composite resin type significantly affected the $\Delta E^{*}$ values of composite resins $(p<0.05)$. There was also significant interaction between polishing method and composite resin type for $\Delta E^{*}$ $(p<0.05)$.

After accelerated aging, all composite resin types showed color changes above the level considered clinically acceptable $\left(\Delta E^{*}{ }_{a b} \leq 3.3\right)^{18)}$. Table 3 shows the mean and standard deviation values of color changes $\left(\Delta E^{*}\right)$ according to composite resin type and polishing method. When the composite resins were evaluated independently, the microhybrid resin showed the lowest $\Delta E^{*}$ values $(p<0.05)$ and the ormocer resin showed the highest $(p<0.05)$. When the effects of polishing method were compared, the polishing wheel (Enhance) showed the highest $\Delta E^{*}$ values and the glaze material (BisCover LV) showed the lowest. There were significant differences between BisCover LV and the other polishing methods $(p<0.05)$, but there were no significant differences among Sof-Lex (polishing disk), Enhance, and the control group $(p>0.05)$.

Composite materials were further compared according to the effects of different polishing methods (Table 3). For Filtek Z250, lowest $\Delta E^{*}$ was obtained with BisCover LV $(p<0.05)$. There were no significant differences among the other polishing methods. For Admira, lowest $\Delta E^{*}$ was obtained with BisCover LV and the highest with Enhance $(p<0.05)$. There were no

Table 2 Two-way ANOVA results for comparison of $\Delta E^{*}, \Delta L^{*}, \Delta a^{*}$, and $\Delta b^{*}$ values

\begin{tabular}{lcccccc}
\hline & Source & Type III Sum of Squares & Df & Mean square & F & Sig. \\
\hline \multirow{2}{*}{$\begin{array}{l}\Delta E^{*} \\
\text { values }\end{array}$} & Composite resin & 240.407 & 3 & 80.136 & 4.378 & 0.000 \\
& Polishing technique & 1.634 .836 & 3 & 544.945 & 29.772 & 0.000 \\
& Composite resin $\times$ Polishing technique & 1.766 .612 & 9 & 196.29 & 10.724 & 0.000 \\
$\Delta L^{*}$ & Composite resin & 2814.304 & 3 & 938.101 & 59.271 & 0.000 \\
values & Polishing technique & 6836.193 & 3 & 2278.731 & 143.974 & 0.000 \\
& Composite resin $\times$ Polishing technique & 2005.888 & 9 & 222.876 & 14.082 & 0.000 \\
\multirow{2}{*}{$\Delta a^{*}$} & Composite resin & 753.108 & 3 & 251.036 & 21.285 & 0.000 \\
values & Polishing technique & 358.947 & 3 & 119.649 & 10.145 & 0.000 \\
& Composite resin $\times$ Polishing technique & 1353.089 & 9 & 150.343 & 12.747 & 0.000 \\
\multirow{2}{*}{$\Delta b^{*}$} & Composite resin & 371.037 & 3 & 123.679 & 8.224 & 0.000 \\
values & Polishing technique & 475.518 & 3 & 158.506 & 10.54 & 0.000 \\
& Composite resin $\times$ Polishing technique & 1448.873 & 9 & 160.986 & 10.705 & 0.000 \\
\hline
\end{tabular}

Table 3 Mean and standard deviation values of color changes $\left(\Delta E^{*}\right)$ of composite resins after accelerated aging

\begin{tabular}{ccccc}
\hline & Filtek P60 & Filtek Z250 & Admira & Grandio \\
\hline Sof-Lex & $24.45(1.56)^{B b}$ & $24.32(1.13)^{A b}$ & $30.71(1.31)^{A a}$ & $22.92(2.31)^{A B b}$ \\
BisCover LV & $15.75(4.03)^{C a}$ & $14.92(3.46)^{B a}$ & $15.15(1.46)^{C a}$ & $20.78(3.45)^{B b}$ \\
Enhance & $31.23(5.43)^{A a}$ & $24.07(1.91)^{A b}$ & $31.26(2.25)^{A a}$ & $21.81(2.13)^{A B b}$ \\
Control & $24.03(1.98)^{B a}$ & $23.61(1.85)^{A a}$ & $25.82(3.54)^{B a}$ & $24.45(3.03)^{A a}$ \\
\hline
\end{tabular}

Same lowercase letters indicate insignificant difference within the same row $(p<0.05)$.

Same uppercase letters indicate insignificant difference within the same column $(p<0.05)$. 
significant differences between Sof-Lex and Enhance. For Filtek P60, lowest $\Delta E^{*}$ was obtained with BisCover $\mathrm{LV}$ and the highest with Enhance $(p<0.05)$. For Grandio, lowest $\Delta E^{*}$ was obtained with BisCover $\mathrm{LV}$ and the highest was obtained in the control group $(p<0.05)$. There were no significant differences between Sof-Lex and Enhance.

Characterization of color changes in CIE-Lab color space

Two-way ANOVA results for $\Delta L^{*}, \Delta a^{*}$, and $\Delta b^{*}$ values of composite resins groups are shown in Table 2. For the $L^{*}$ value, there were significant differences both among composite resin types and polishing methods $(p<0.05)$. For all groups, their $L^{*}$ values decreased after accelerated aging $\left(\Delta L^{*}<0\right)$ - that is, the specimens became darker. When the effects of composite resin type were compared, lowest $\Delta L^{*}$ was observed in Filtek P60 and the highest in Admira. When the effects of polishing method were compared, lowest $\Delta L^{*}$ was observed in BisCover LV and the highest in the control group.

The $a^{*}$ values increased after accelerated aging for all groups $\left(\Delta a^{*}>0\right)$ - that is, the specimens shifted toward red. There were significant differences among composite resin types and polishing methods $(p<0.05)$. When the effects of composite resin type were compared, lowest $\Delta a^{*}$ was observed in Admira and the highest in Filtek P60 $(p<0.05)$. When the effects of polishing method were compared, lowest $\Delta a^{*}$ was observed in the control group and the highest in Enhance.

The $b^{*}$ values decreased after accelerated aging for all groups $\left(\Delta b^{*}<0\right)$ - that is, the specimens shifted toward blue. There were significant differences among composite resin types and polishing methods $(p<0.05)$.
When the effects of composite resin type were compared, lowest $\Delta b^{*}$ was observed in Filtek Z250 and the highest in Filtek P60 $(p<0.05)$. When the effects of polishing method were compared, lowest $\Delta b^{*}$ was observed in BisCover LV and the highest in Enhance.

\section{Surface roughness changes}

Table 4 shows the surface roughness $(R a)$ values before and after accelerated aging according to composite resin type and polishing method. The $R a$ values of the control groups decreased after aging in all composite groups $(p<0.05)$. On the contrary, the $R a$ values of Filtek P60/ BisCover LV and Filtek Z250/Sof-Lex groups increased after aging $(p<0.05)$. For the other remaining groups, there were no significant differences in $R a$ value before and after aging $(p>0.05)$.

\section{SEM images of surface topography}

The microhybrid composite resin (Filtek Z250) showed the lowest $\Delta E^{*}$ values and the ormocer composite resin (Admira) showed the highest. The SEM images of Filtek Z250 and Admira before and after accelerated aging and according to polishing method are shown in Figs. 1-4 and Figs. 5-8 respectively.

SEM analysis showed good agreement with our color change data. SEM images of composite resins polished by BisCover LV glaze material showed the smoothest surfaces (Figs. 2(a) and (b), Figs. 6(a) and (b)) before and after accelerated aging.

For Filtek Z250, SEM images showed slight differences in surface topography before and after aging for each polishing method (Figs. 1-3), except for the control group (Fig. 4). Among the polishing methods, BisCover LV glaze material resulted in the smoothest

Table $4 R a$ values $(\mu \mathrm{m})$ expressed in mean value (standard deviation) before and after aging for each composite resin and polishing method

\begin{tabular}{|c|c|c|c|c|}
\hline & & Before aging & & \\
\hline \multirow{4}{*}{ Grandio } & Enhance & $0.64(0.12)$ & $0.76(0.20)$ & $(p=0.29)$ \\
\hline & Sof-Lex & $0.29(0.04)$ & $0.55(0.27)$ & $(p=0.06)$ \\
\hline & BisCover LV & $0.60(0.42)$ & $0.60(0.26)$ & $(p=0.80)$ \\
\hline & Control & $1.39(0.24)$ & $0.67(0.17)$ & $(p=0.001)$ \\
\hline \multirow{4}{*}{ Admira } & Enhance & $0.62(0.17)$ & $0.88(0.49)$ & $(p=0.29)$ \\
\hline & Sof-Lex & $0.26(0.11)$ & $0.52(0.21)$ & $(p=0.05)$ \\
\hline & BisCover LV & $0.51(0.26)$ & $0.62(0.09)$ & $(p=0.39)$ \\
\hline & Control & $1.33(0.21)$ & $0.67(0.19)$ & $(p=0.001)$ \\
\hline \multirow{4}{*}{ Filtek P60 } & Enhance & $0.62(0.16)$ & $0.70(0.28)$ & $(p=0.61)$ \\
\hline & Sof-Lex & $0.45(0.05)$ & $0.55(0.17)$ & $(p=0.22)$ \\
\hline & BisCover LV & $0.49(0.07)$ & $2.59(1.21)$ & $(p=0.005)$ \\
\hline & Control & $1.45(0.26)$ & $0.42(0.14)$ & $(p=0.001)$ \\
\hline \multirow{4}{*}{ Filtek Z250 } & Enhance & $0.41(0.04)$ & $0.50(0.17)$ & $(p=0.28)$ \\
\hline & Sof-Lex & $0.18(0.03)$ & $0.67(0.09)$ & $(p=0.001)$ \\
\hline & BisCover LV & $0.56(0.29)$ & $0.71(0.15)$ & $(p=0.32)$ \\
\hline & Control & $1.22(0.17)$ & $0.56(0.17)$ & $(p=0.001)$ \\
\hline
\end{tabular}

Statistical significant is set at $\alpha=0.05$. 

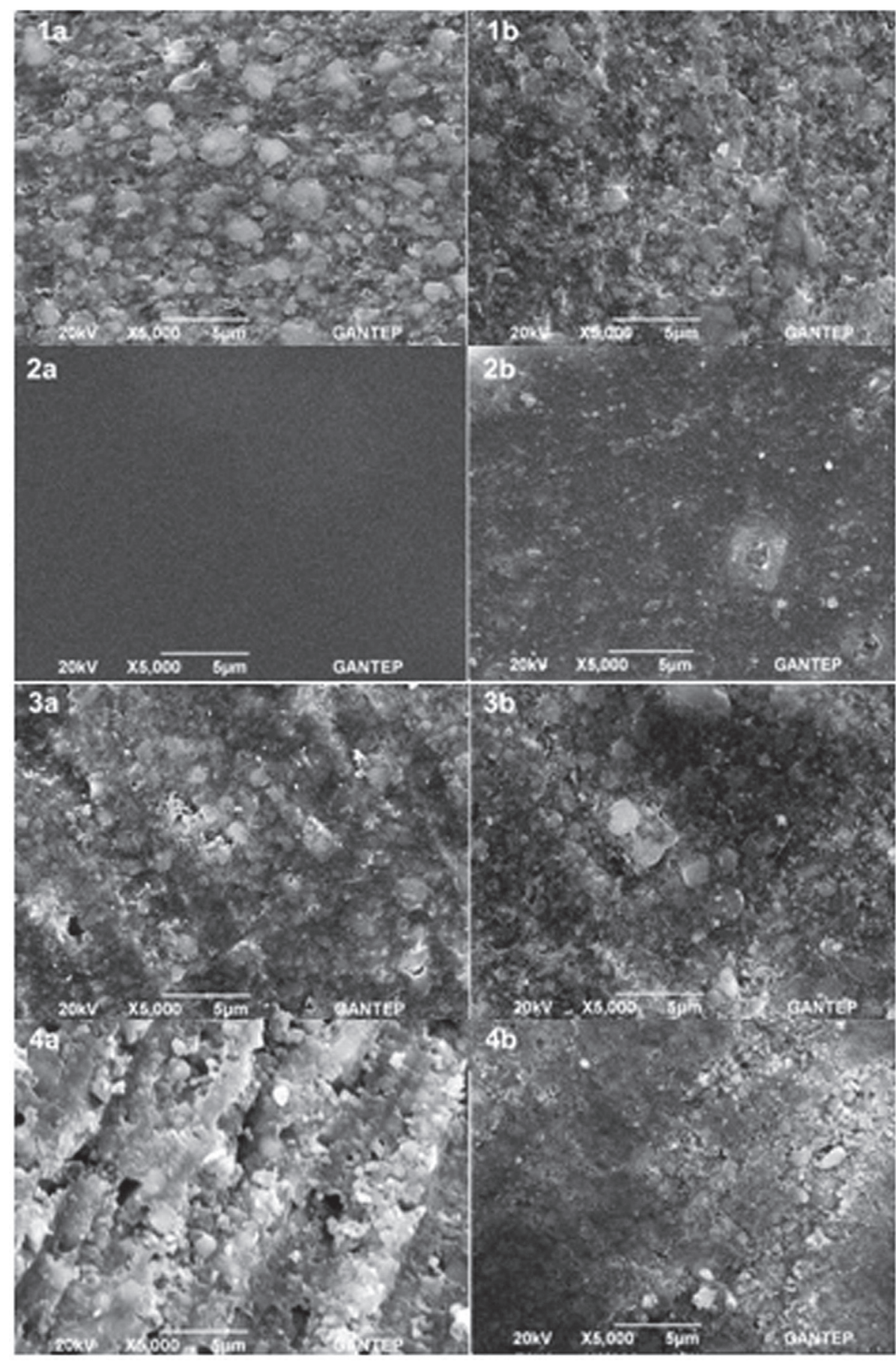

Fig. 1-4 SEM images of Filtek Z250 before aging (a) and after aging (b) and polished by: (1) Sof-Lex, (2) BisCover LV, (3) Enhance, and (4) Control.

surfaces (Figs. 2(a) and (b)).

For Admira, surface differences before and after aging could be clearly seen in the SEM photographs. Polishing with Sof-Lex resulted in a rough surface and protrusion of filler particles after accelerated aging (Figs. 5(a) and (b)). Similarly, polishing by Enhance resulted in the exposure of more filler particles after accelerated aging (Figs. 7(a) and (b)).

\section{DISCUSSION}

This in vitro study compared the effects of different polishing methods on the color stability of four composite resin materials —with different filler contents and 


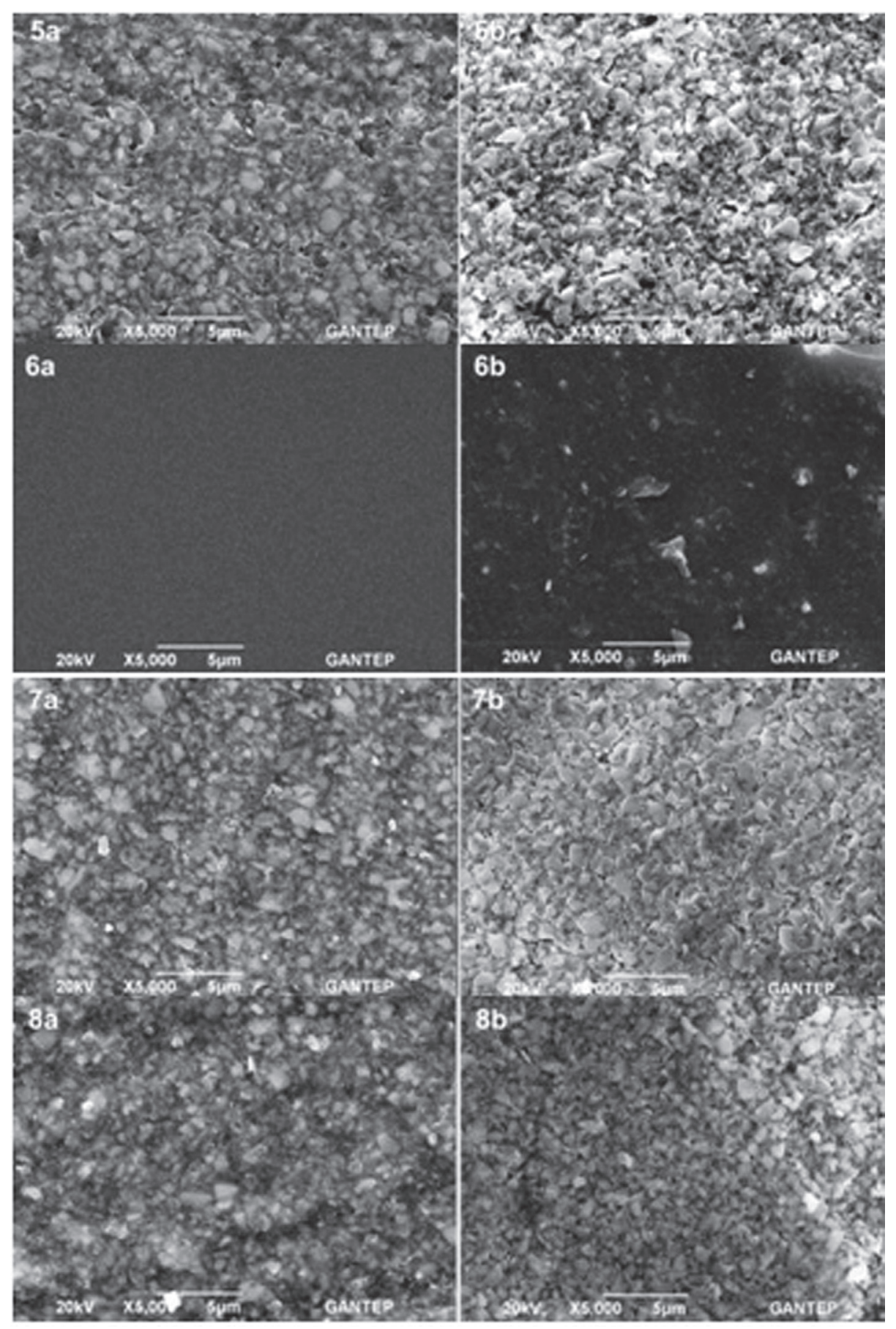

Fig. 5-8 SEM images of Admira before aging (a) and after aging (b) and polished by: (5) Sof-Lex, (6) BisCover LV, (7) Enhance, and (8) Control.

sizes - after accelerated aging. When comparing the effects of composite resin types, the microhybrid resin showed the lowest $\Delta E^{*}$ values and the ormocer resin the highest. There was also significant interaction between composite type and polishing method for $\Delta E^{*}$, such that the highest and lowest $\Delta E^{*}$ values were observed in Admira/Enhance and Filtek Z250/BisCover LV groups respectively. Therefore, the null hypothesis of this study was rejected because the color stability of composite resins was affected by the different polishing methods and composite types.

Factors affecting the color stability of resin composites The color stability of composite resins is influenced by 
both extrinsic and intrinsic factors. Extrinsic factors include the duration and intensity of light emission during the curing process, exposure to environmental factors such as ambient and ultraviolet radiation, water, heat, and food colorants. Intrinsic factors include composition of the resin matrix, filler loading and particle size distribution, type of photoinitiator, and percentage of remaining $\mathrm{C}=\mathrm{C}$ bonds ${ }^{26}$.

Composite resin restorations must harmonize in shape and shade with the adjacent natural teeth: they must not only function properly but also fulfill aesthetic demands. However, the human perception of colors is affected by external factors ${ }^{25)}$. Light transmittance is an optical property of materials and an important factor which influences color selection ${ }^{27)}$. A difference in the light transmittance property of dental resin composites can affect their clinical appearance. Specifically in the case of dental composites, their light transmittance property is affected by the background color. When light is illuminated on a translucent specimen backed by a white background, many fractions of light reaching the background would be reflected ${ }^{28)}$. Therefore, a white plate was used for the background in this study, as was thus done in other published studies ${ }^{21-23)}$.

Relation between color stability and surface roughness of resin composites

Optical properties of dental resin composites are directly affected by surface roughness ${ }^{28)}$. An increasingly roughened surface will reflect the individual segments of specular beam at slightly different angles ${ }^{29)}$. In the current study, the ormocer resin (Admira) showed the highest $\Delta E^{*}$ values. These color change results were supported by evidence of SEM images of Admira's clearly roughened surfaces after accelerated aging (Figs. 5-8). Notably, polishing with Sof-Lex exacerbated surface roughness with protrusion of coarse filler particles, especially after aging (Figs. 5(a) and (b)).

Except for the control group of each composite resin type, there were no significant differences in surface roughness before and after aging for all the three polishing methods applied to each composite resin. In all the control groups, no finishing or polishing was performed on the composite specimens. However, when polishing was applied, filler particles on composite resin surfaces were easily abraded by the polishing device, thus contributing to the surface finish of polished specimens. In the clinical setting, the advantages of polishing composite resin restorations prevail during the aging process until it is completed. Polishing reduces stain and plaque accumulation and provides better color stability.

Combined effect of composite resin type and polishing method on color stability

An ormocer matrix (Admira) is characterized by an interpenetrating network of inorganic-organic copolymers. The resin matrix and inorganic filler differ in hardness, and they cannot be uniformly abraded. Nonetheless, the ormocer matrix exhibits significantly less wear than the composite matrices ${ }^{30)}$. Interestingly, the ormocer resin showed the highest $\Delta E^{*}$ values in the current study - an outcome stemming from the significant and inextricable interaction between polishing method and the highly crosslinkable organic network and inorganic components of ormocers.

On the other hand, the microhybrid resin (Filtek Z250) showed the lowest $\Delta E^{*}$ values compared to the other composite resins. The low staining susceptibility of Filtek Z250 was most probably linked to its low water sorption rate, which in turn was linked to its hydrophobic matrix $^{31)}$.

Pires-de-Souza Fde et al. ${ }^{32}$ conducted a similar study which evaluated the in situ color stability, surface morphology, and tooth-restoration interface degradation of different light-polymerized composites (hybrid, microhybrid, nanoparticle-reinforced, and siloranebased) after artificial accelerated aging. The siloranebased composite showed the highest color change $\left(\Delta E^{*}=18.6 \pm 2.2\right)$ and the microhybrid resin (Filtek Z250) showed the lowest $\left(\Delta E^{*}=8.66 \pm 2.35\right)$. Similarly in the current study, the color changes of composite resins in all groups were above the clinically acceptable level $\left(\Delta E^{*} \leq 3.3\right)^{18)}$, and that the microhybrid resin (Filtek Z250) showed the lowest $\Delta E^{*}$ values compared to the other composite resins. These results were supported by evidence of SEM images of Filtek Z250 according to each polishing method, which displayed slight differences before and after accelerated aging (Figs. 1-3).

In light of the combined effect of and significant interaction between composite resin type and polishing method on the color stability of resin composites, it is recommended that the polishing of Filtek Z250 and Grandio composites be performed with BisCover LV glaze material (Figs. 2(a) and (b) and Table 3).

\section{Comparison of the effects of different polishing methods}

1. Polishing disks versus polishing wheels

Polishing tools used over the years range from multiplestep systems using fine and superfine diamond burs, aluminum oxide abrasive disks, diamond- and siliconimpregnated rubber cups to one-step polishing systems such as diamond-impregnated cups and silicon carbide brushes ${ }^{33)}$. The efficacy of polishing disks impregnated with aluminum oxide particles to produce smooth surfaces lies in their ability to equally remove both organic matrix and filler particles. While the plane movement of these polishing disks contributes to a smoother surface ${ }^{34)}$, these disks have limitations due to their geometry. They are difficult to produce and when using these disks, it is difficult to polish the anatomically contoured surfaces of dental composite restorations, especially in the posterior region of the mouth.

In contrast, polishing wheels consist of a flexible rubber-like material, a polymerized resin impregnated with an abrasive ${ }^{31)}$. They wear the resin matrix and contour prominent surfaces only, thus resulting in greater surface roughness ${ }^{35)}$ and creating rougher surfaces than polishing disks ${ }^{29)}$.

In the current study, none of the polishing methods 
resulted in significant differences in surface roughness during the aging process, except for Filtek Z250/ Sof-Lex and Filtek P60/BisCover LV groups. These surface roughness results further suggested that the color stability of resin composites was affected by the interaction between composite resin type and polishing method, as seen in the highest $\Delta E^{*}$ value obtained with Enhance.

\section{Glaze materials}

BisCover LV is a low-viscosity, light-cured resin formulation used to seal composite restorations while leaving a smooth polished/glazed surface. It boasts of a slew of both aesthetic and practical advantages: resistant to staining and color shifting, and hence yield better aesthetic results; can be used inside and outside the mouth to finish dental composite restorations; eliminates manual polishing steps; saves chair-time; and reduces the number of polishing products needed in the dental operatory ${ }^{36)}$.

On the influence of surface sealants on color stability, Catelan et al. ${ }^{37)}$ reported that after resin composites were subjected to accelerated aging or prolonged immersion in staining solutions, they all showed some color change and that BisCover LV did not affect their color stability. In another study by Güler et al. ${ }^{38)}$, they investigated the effects of different polishing methods on the color stability of different composite resins upon exposure to a staining agent. Highest color change values were obtained for specimens polished with BisCover LV liquid polishing system, and that these $\Delta E^{*}$ values were significantly higher when compared to the other polishing methods. In the current study, lowest $\Delta E^{*}$ values were obtained with BisCover LV, and that these $\Delta E^{*}$ values were also significantly different from the other polishing methods (Table 3).

On the influence of surface sealants on surface roughness, Attar ${ }^{39)}$ reported that the use of BisCover surface sealant significantly improved the surface smoothness of all tested composites. In the current study, SEM images revealed that Admira and Filtek Z250 showed the smoothest surfaces when applied with BisCover LV glaze material (Figs. 2 and 6).

\section{Effects of polymerization variables on color change}

Various types of light sources have been used to convert composite resins, and studies were carried out to investigate the effects of polymerization variables on the color of dental composite resins: intensity of emitted light, exposure time, type of composite resin, type of initiator, and distance between tooth and curing source ${ }^{40)}$. In the case of visible light-cured composites, polymerization occurs upon exposure to an intense blue light. The visible light is absorbed by a diketone ( $\alpha$-diketone), which in the presence of an organic amine initiator, starts the polymerization reaction ${ }^{41)}$. In a study by Setz et $a l .{ }^{42)}$, it was found that composites with a high filler content showed better color stability, and vice versa. Nonetheless, no strong correlation was found between filler content and color stability ${ }^{42)}$.
Effects of accelerated aging on color change

Paravina et al ${ }^{43)}$ reported that accelerated aging generally caused a decrease in $L^{*}$ (specimens became darker) and $a^{*}$ (specimens become redder) values, but an increase in $b^{*}$ value (specimens became more saturated/chromatic). In the present study, accelerated aging caused a color shift from yellow to blue $\left(\Delta b^{*}<0\right)$ and from green to red $\left(\Delta a^{*}>0\right)$. Specimens also became darker $\left(\Delta L^{*}<0\right)$ after accelerated aging.

Methodological limitations are inherent to all in vitro studies. In the present study, artificial accelerated aging was used to simulate the effects of long-term exposure in an oral environmental within a short time period, with an aim to anticipate the clinical performance of dental composite resins in terms of color stability. In the oral environment, however, saliva and other fluids may dilute the stains. Besides the presence of water, temperature changes and $\mathrm{pH}$ level in the oral cavity may affect the properties of dental composite restorations. It should also be mentioned that in this study, flat specimen surfaces were used; clinically, composite resin restorations have an irregular geometric structure of convex and concave surfaces.

\section{CONCLUSIONS}

Within the limitations of this study, the following conclusions were drawn:

1. Composite resin type and polishing method significantly affected the color stability of composite resins after accelerated aging.

2. Polishing by BisCover LV glaze material resulted in the lowest color change.

3. For all composite resin types, accelerated aging caused a decrease in their $L^{*}$ values (specimens became darker), an increase in their $a^{*}$ values (specimens shifted toward red), and a decrease in their $b^{*}$ values (specimens shifted toward blue).

4. Except for Filtek Z250/Sof-Lex and Filtek P60/ BisCover LV groups, no significant differences in surface roughness were observed for the other experimental groups after accelerated aging.

\section{ACKNOWLEDGMENTS}

The authors of this study would like to thank Ziya Gesoğlu (Physical Engineering Department, Gaziantep University) for his kind assistance with the accelerated aging device.

\section{REFERENCES}

1) Carlsson GE, Wagner IV, Ödman P, Ekstrand K, MacEntee M, Marinello C, Nanami T, Ow RK, Sato H, Speer C, Strub JR, Watanabe T. An international comparative multicenter study of assessment of dental appearance using computer aided image manipulation. Int J Prosthodont 1998; 11: 246254.

2) Davis LG, Ashworth PD, Spriggs LS. Psychological effects of aesthetic dental treatment. J Dent 1998; 26: 547-554.

3) Puckett AD, Fitchie JG, Kirk PC, Gamblin J. Direct composite 
restorative materials. Dent Clin North Am 2007; 51: 659675.

4) Taira M, Okazaki M, Takahashi J. Studies on optical properties of two commercial visible-light-cured composite resins by diffuse reflectance measurements. J Oral Rehabil 1999; 26: 329-337.

5) Kim IJ, Lee YK. Changes in color and color parameters of dental resin composites after polymerization. J Biomed Mater Res B Appl Biomater 2007; 80: 541-546.

6) Uchida H, Vaidyanathan J, Viswanadhan T, Vaidyanathan TK. Color stability of dental composites as a function of shade. J Prosthet Dent 1998; 79: 372-377.

7) Stober T, Gilde H, Lenz P. Color stability of highly filled composite resin materials for facings. Dent Mater 2001; 17: 87-94.

8) Lee YK, El Zawahry M, Noaman KM, Powers JM. Effect of mouthwash and accelerated ageing on the color stability of esthetic restorative materials. Am J Dent 2000; 13: 159-161.

9) Yap AU, Mok BY. Surface finish of a new hybrid aesthetic restorative material. Oper Dent 2002; 27: 161-166.

10) Dunkin RT, Chambers DW. Gingival response to class V composite resin restorations. J Am Dent Assoc 1983; 106: 482-484.

11) Vichi A, Ferrari M, Davidson LC. Color and opacity variations in three different resin-based composite products after water aging. Dent Mater 2004; 20: 530-534.

12) Reis AF, Giannini M, Lovadino JR, dos Santos Dias CT. The effect of six polishing systems on the surface roughness of two packable resin-based composites. Am J Dent 2002; 15: 193197.

13) Buchalla W, Attin T, Hilgers R, Hellwig E. The effect of water storage and light exposure on the color and translucency of a hybrid and a microfilled composite. J Prosthet Dent 2002; 87: 264-270.

14) Dietschi D, Campanile G, Holz J, Meyer JM. Comparison of the color stability of ten new-generation composites: an in vitro study. Dent Mater 1994; 10: 353-362.

15) Paul SJ, Peter A, Rodoni L, Pietrobon N. Conventional visual vs spectrophotometric shade taking for porcelain-fusedto-metal crowns: a clinical comparison. Int $J$ Periodontics Restorative Dent 2004; 24: 222-231.

16) Culpepper WD. A comparative study of shade-matching procedures. J Prosthet Dent 1970; 24: 166-173.

17) Hosoya Y, Goto G. Color changes of light-cured composite resins. J Clin Pediatr Dent 1992; 16: 247-252.

18) Ruyter IE, Nilner K, Moller B. Color stability of dental composite resin materials for crown and bridge veneers. Dent Mater 1987; 3: 246-251.

19) Davis BA, Friedl KH, Powers JM. Color stability of hybrid ionomers after accelerated aging. J Prosthodont 1995; 4: 111115.

20) Yap AU, Han VT, Soh MS, Siow KS. Elution of leachable components from composites after LED and halogen light irradiation. Oper Dent 2004; 29: 448-453.

21) Tak O, Altintas SH, Ozturk N, Usumez A. Effect of tree types of light-curing units on 5-year colour changes of light-cured composite. Clin Oral Investig 2009; 13: 29-35.

22) Kim SH, Lee YK. Changes in color and color coordinates of an indirect resin composite during curing cycle. J Dent 2008; 36: 337-342.

23) Kim BJ, Yu B, Lee YK. Shade distribution of indirect resin composites compared with a shade guide. J Dent 2008; 36 : 1054-1060.

24) Inokoshi S, Burrow MF, Kataumi M, Yamada T, Takatsu T. Opacity and color changes of tooth-colored restorative materials. Oper Dent 1996; 21: 73-80.

25) Knispel G. Factors affecting the process of color matching restorative materials to natural teeth. Quintessence Int 1991; 22: 525-531.

26) Sarafianou A, Iosifidou S, Papadopoulos T, Eliades G. Color stability and degree of cure of direct composite restoratives after accelerated aging. Oper Dent 2007; 32: 406-411.

27) Brodbelt RH, O'Brien WJ, Fan PL. Translucency of dental porcelains. J Dent Res 1980; 59: 70-75.

28) Lee YK, Lim BS, Kim CW. Effect of surface conditions on the color of dental resin composites J Biomed Mater Res 2002; 63: 657-663.

29) Sarac D, Sarac YS, Kulunk S, Ural C, Kulunk T. The effect of polishing techniques on the surface roughness and color change of composite resins. J Prosthet Dent 2006; 96: 33-40.

30) Kunzelmann KH, Mehl A, Hickel R. Sliding wear of an experimental ormocer and 15 commercial composites. J Dent Res 1998; 77: Abstract No. 965.

31) Douglas WH, Craig RG. Resistance to extrinsic stains by hydrophobic composite resin systems. J Dent Res 1982; 61: 41-43.

32) Pires-de-Souza Fde C, Garcia Lda F, Roselino Lde M, Naves LZ. Color stability of silorane-based composites submitted to accelerated artificial ageing —an in situ study. J Dent 2011; 39 Suppl 1: e18-24.

33) St-Georges AJ, Bolla M, Fortin D, Muller-Bolla M, Thompson JY, Stamatiades PJ. Surface finish produced on three resin composites by new polishing systems. Oper Dent 2005; 30: 593-597.

34) Ritter AV. Posterior resin-based composite restorations: clinical recommendations for optimal success. J Esthet Restor Dent 2001; 13: 88-89.

35) Turkun LS, Turkun M. The effect of one-step polishing system on the surface roughness of three esthetic resin composite materials. Oper Dent 2004; 29: 203-211.

36) http://www.bisco.com/catalog/biscoverlv.pdf, 19.08.2011-14:15

37) Catelan A, Briso AL, Sundfeld RH, Goiato MC, dos Santos $\mathrm{PH}$. Color stability of sealed composite resin restorative materials after ultraviolet artificial aging and immersion in staining solutions. J Prosthet Dent 2011; 105: 236-241.

38) Güler AU, Güler E, Yücel AC, Ertas E. Effects of polishing procedures on color stability of composite resins. J Appl Oral Sci 2009; 17: 108-112.

39) Attar N. The effect of finishing and polishing procedures on the surface roughness of composite resin materials. J Contemp Dent Pract 2007; 8: 27-35.

40) Lee YK, Lim BS, Kim CW. Influence of illuminating and viewing aperture size on the colour of dental resin composites. Dent Mater 2004; 20: 116-123.

41) Craig RG, Powers JM. Restorative dental materials. 11th ed. St. Louis: Mosby; 2002.

42) Setz J, Lin W, Pfeifer H. Color stability of light curing composites. Dtsch Zahnarztl Z 1990; 45: 666-668.

43) Paravina RD, Ontiveros JC, Powers JM. Accelerated aging effects on color and translucency of bleaching-shade composites. J Esthet Restor Dent 2004; 16:117-126. 